\title{
Pharmacological Inhibition of Focal Adhesion Kinase Attenuates Cardiac Fibrosis in Mice Cardiac Fibroblast and Post-Myocardial-Infarction Models
}

\author{
Guang-Pu Fan ${ }^{\mathrm{a}}$ Wei Wang ${ }^{\mathrm{a}}$ Hui Zhao ${ }^{\mathrm{a}}$ Lin Cai $^{\mathrm{b}}$ Pei-De Zhang ${ }^{\mathrm{a}}$ Zi-He Yang ${ }^{\mathrm{b}}$ \\ Jing Zhang ${ }^{\mathrm{a}} \mathrm{Xu}$ Wang $^{\mathrm{a}}$ \\ ${ }^{a}$ Cardiovascular Surgery Department, FuWai Hospital\& Cardiovascular Institute, Peking Union Medical \\ College\& Chinese Academy of Medical Sciences, Beijing, bState Key Laboratory of Cardiovascular \\ Disease, Fuwai Hospital, National Center for Cardiovascular Diseases, Chinese Academy of Medical \\ Sciences and Peking Union Medical College, Beijing, P. R. China
}

\section{Key Words}

Focal adhesion kinase - Cardiac fibroblasts - Cardiac fibrosis - PF-562,271 - Myocardial infarction

\begin{abstract}
Background: To investigate the role of focal adhesion kinase (FAK)-mediated signaling in hypoxia-induced cardiac fibroblasts (CFs) differentiation and cardiac fibrosis post-myocardial infarction (MI) on a mice model. Methods: CFs of neonatal C57BL/6 mice were treated under normoxic, hypoxic, or hypoxic+PP2 (known as a Src kinase family inhibitor) conditions. Gene expressions of FAK, alpha-smooth muscle actin ( $\alpha$-SMA) and collagen type I alpha 1 (Col $1 \alpha 1)$, or $\alpha-S M A$ and vimentin levels were performed by RT-PCR and immunofluorescence staining, respectively. Thirty mice were surgically treated into Sham $(n=7)$ and MI $(n=23)$ groups; and FAK inhibitor PF-562271 was given to six survivor MI mice (as PF group, from 15 survivors). Heart function and collagenous tissues were examined by echocardiography, as well as by Masson's trichrome and Sirius red staining, respectively. Type I collagen, FAK protein, mTOR, ERK1/2, AKT, P70S6K and phospho-FAK levels were also analyzed. Results: FAK inhibition with PP2 significantly decreased CFs differentiation and collagen synthesis under hypoxia treatment. In vivo, PF-562271 treatment resulted in fibrosis attenuation; however, deteriorated heart function of MI mice could not be significantly improved. PF-562271 may affect phosphomTOR $(p<0.05)$, phospho-ERK1/2 $(p<0.01)$, phospho-AKT $(p<0.001)$ and phospho-P70S6K $(p<0.05)$ to exert its benefits. FAK can be activated either under hypoxia in CFs or MI in a mouse model to promote fibrosis. However, pharmacological inhibition of FAK can attenuate fibrosis response. Conclusion: This study provides novel evidence that FAK inhibition may become a promising pharmaceutical strategy to attenuate fibrosis post-MI.
\end{abstract}

Copyright $@ 2015$ S. Karger AG, Basel

Wei Wang,

KARGER 125
Cardiovascular Surgery Department, FuWai Hospital\& Cardiovascular Institute, Peking Union Medical College\& Chinese Academy of Medical Sciences, 167 Bei Li Shi Rd, Beijing, 100037, (P. R. China); E-Mail drweiwang0728@hotmail.com 


\section{Introduction}

Cardiac remodeling is an important pathological consequence with its dynamic and progressive features [1]. Fibrosis in post-myocardial infarction (MI), seen in both infarcted and non-infarcted myocardium, progressively impairs ventricular function and leads to poor prognosis $[2,3]$. Despite a relatively sophisticated understanding of the cell biological processes of fibrosis development, there are still few effective strategies to prevent it; emphasizing a need for new insights into this disorder.

Focal adhesion kinase (FAK) is a 125 -kDa non-receptor cytoplasmic tyrosine kinase, which is known to play a pivotal role in regulating cell migration, proliferation and survival in a range of cell types [4-6]. FAK has recently received attention as a potential mediator of fibrosis. Classically, FAK could mediate fibroblast attachment to extracellular matrix (ECM). Moreover, it is also involved in transducing signals from growth factors [7]. Previous studies have demonstrated that FAK downstream signaling is involved in two primary pathways that involve fibrosis [8-11]. Phosphatidylinositol 3-kinase/protein kinase B (PI3K/AKT) signaling pathway is known as a target of rapamycin/S6 kinase (mTOR/S6K) complex, while extracellular signal-regulated kinase $1 / 2$ (ERK1/2) signaling pathway is one of mitogenactivated protein kinase (MAPK) signaling pathways; both play a role in c-Jun N-terminal kinase (JNK) phosphorylation. Previous studies in animal models have shown various FAK mechanisms and functionalities such as in the lungs and heart, with contradiction [7]. FAK plays a critical role in myocyte survival and increases cardiac fibrosis during hypertrophy or ischemia/reperfusion (I/R) period in myocyte-restricted FAK conditional knockout mice $[8,9]$. Fibrosis attenuations and collagen content in overloaded left ventricle were observed by siRNA-induced-myocardial FAK silencing [10]; and enhanced cardiac FAK activity could not induce cardiac fibrosis from I/R injury [12]. Therefore, a comprehensive investigation is warranted on such an important active molecule in fibrosis formation.

We previously reported $[13,14]$ that FAK is involved in atrial fibrosis, and FAK inhibition suppresses $\alpha$-SMA expressions in TGF $\beta 1$-induced fibroblasts, which is crucial in the appearance of cardiac fibrosis. Therefore, it seems reasonable to postulate that FAK inhibition could be a therapeutic target in counteracting pro-fibrotic mechanisms, given that fact that FAK participates in the pathophysiology of fibrosis.

In the present study, we used primary cardiac fibroblasts (CFs) and established an MI animal model of cardiac fibrosis to test our hypothesis that FAK participates in the processes of hypoxia induced- and post-MI cardiac fibrosis in vitro and in vivo, respectively. In addition, pharmacologic inhibition of FAK on cardiac fibrosis with its regulation of relevant signaling pathways was investigated.

\section{Materials and Methods}

The present study with its experimental design was approved by the Ethics Committee of the Chinese Academy of Medical Sciences and Peking Union Medical College (No. 201304-M-90-YJSH). Animal care and experimental procedures were conducted in accordance with the European Guidelines on Laboratory Animal Care. Eight- to ten-week-old C57BL/6 wild-type mice (male only) were used in this study. Animals were housed in temperature-controlled facilities on a 12-hour light/dark cycle with normal diet.

MI was induced by permanent left anterior descending (LAD) coronary artery ligation (CAL). Mice were anesthetized by intraperitoneally administering $320( \pm 40) \mathrm{mg} / \mathrm{kg}$ of tribromoethanol $(20 \mathrm{mg} / \mathrm{ml}$ in saline solution). Surgical procedures were as follows: briefly, after intubation, left lateral thoracotomy was performed at the fourth intercostal space; a 7-0 polypropylene suture was then placed around the left anterior descending artery just below the atrioventricular border. Ischemia was evident from discoloration of the infarct artery-related area. As non-infarcted controls, mice underwent sham operation where the ligature around the LAD was not tied. Animals were recovered from anesthesia under warm conditions with normal ventilation.

Four weeks after MI, animals were sacrificed and hearts were harvested for further analysis. To evaluate cardiac function and morphology, echocardiography was performed before tissues were harvested. 
Pharmacological approach that specifically targets the fibrogenic process was introduced with PF562271 (obtained from Selleck Chemicals, Houston, TX); which is a novel, ATP-competitive, reversible FAK (and Pyk2) inhibitor. At the $7^{\text {th }}$ day of post-MI, an employed dosage of PF-562271 (15 mg/kg body weight in $0.2 \mathrm{ml}$ of $0.5 \%$ methylcellulose) was orally given to experimental animals on a daily basis over a period of 21 days.

Echocardiography of sedated mice was carried out using a Vevo 770 high resolution imaging system (VisualSonics, Toronto, Canada) equipped with a $40 \mathrm{MHz}$ transducer. Three independent M-mode measurements per animal were performed by an experienced examiner. Echocardiographic data were recorded at heart rates between 450 and $550 \mathrm{BPM}$. The following parameters were obtained in the short axis at the papillary muscle level: end systolic and end diastolic chamber diameters, and left ventricular fractional shortening. An anatomic M-mode measurement would be chosen when the heart was rotated at a position of adhesion to the chest wall, to reveal the real diameter.

CFs were isolated from hearts of two- to four-day-old neonatal C57BL/6 mice and cultured as described previously [15]. Briefly, the heart was sliced and tissues went through enzymatic digestion. Myocardial cells were wiped off; and primary cells were purified and maintained in Dulbecco's Modified Eagle's Medium (DMEM, Invitrogen) containing 10\% fetal bovine serum (Invitrogen), penicillin (100 U/ $\mathrm{ml})$ and streptomycin $(100 \mu \mathrm{g} / \mathrm{ml})$. Trypsin-EDTA solution $(0.05 \%)$ was used for sub-culturing fibroblasts and third-generation cells were used for experiments. Cells were starved for three hours prior to treatment.

FAK/Src inhibitor PP2 (Calbiochem, USA) was chosen in the in vitro experiments based on its solubility and our previous report [15]. CFs were treated as follows: first, according to our previous protocol on hypoxia treatment [16], cells in hypoxia and hypoxia+PP2 groups were cultured in a hypoxic GENbox jar fitted with a catalyst (Bio-Me'rieux) to scavenge free oxygen; second, cells were pretreated for one hour and with PP2 $(5 \mu \mathrm{M})$ for 24 hours before hypoxia was applied; third, cells were cultured in dishes or glass coverslips for RT-PCR analysis or immunofluorescence staining, respectively.

Cells grown on glass coverslips were stained as described previously [15]. Briefly, fibroblasts were rinsed with PBS and fixed in 4\% formaldehyde for 10 minutes; then, washed three times in PBS and permeated with $0.2 \%$ Triton X-100 for 10 minutes. Washing was repeated and coverslips were blocked by $5 \%$ BSA blocking buffer for 30 minutes. Anti- $\alpha$-SMA (1:400, Abcam, Hong Kong, China) and Anti-vimentin (1:1000, Abcam, Hong Kong, China) were applied to cells; then, cells were incubated overnight at $4{ }^{\circ} \mathrm{C}$. Coverslips were washed three times in PBS and treated with secondary antibodies for 45 minutes at room temperature; anti- $\alpha$-SMA and anti-vimentin were labeled with fluorescein (FITC)-conjugated IgG (1:400, Santa Cruz) or rhodamine (TRITC)-conjugated IgG (1:400, Santa Cruz), respectively. Nuclei was stained by 4',6-diamidino-2-phenylindole (DAPI). After slides were mounted in glycerol, cells were analyzed under a Leica TCS-SP5 Confocal Laser Scanning Microscope (Leica, Germany).

Total RNA of CFs was extracted with Trizol. Briefly, extracted samples (1-2 $\mu \mathrm{g})$ were reverse transcribed (Fermentas k1622 kit); and cDNA samples were amplified by PCR (KAPA SYBR $®$ rapid quantitative PCR kit, KK4600) using specific primers against FAK (sense, 5'-TCC ACC AAA GAA ACC ACC TC-3'; antisense, 5'-ACG GCT TGA CAC CCT CAT T-3'), $\alpha$-SMA (sense, 5'-AGG GAG TGA TGG TTG GAA TG-3'; antisense, 5'-GAT GAT GCC GTG TTC TAT CG-3') and Col1 $\alpha 1$ (sense, 5'-ATC TCC TGG TGC TGA TGG AC-3'; antisense, 5'-GCC TCT TTC TCC TCT CTG ACC-3'). GAPDH (sense, 5'-ACA GCA ACA GGG TGG TGG AC-3'; antisense, 5'-TTT GAG GGT GCA GCG AAC TT-3') was used as loading control. The procedure was carried out under the following conditions: incubation at $95{ }^{\circ} \mathrm{C}$ for three minutes, followed by denaturation at $95^{\circ} \mathrm{C}$ for three minutes; then, annealing and extension at $60^{\circ} \mathrm{C}$ for 20 seconds. A negative control was also included in each experiment.

Hearts were harvested, weighed, washed in phosphate-buffered saline, fixed in $4 \%$ paraformaldehyde overnight and embedded in paraffin. For Masson's trichrome staining (Leagene, Beijing, China.) or Sirius red staining (Leagene, Beijing, China.), each paraffin-embedded heart was cut into 4 or $8 \mu \mathrm{m}$ thick sections, respectively; and procedures were mainly based on manufacturer's instructions. Each section was imaged under a microscope (Leica, DM600 B). Fibrosis was calculated by computerized planimetry using ImageJ software, version 1.44 (NIH, Bethesda, MD, USA).

Paraffin embedded $4 \mu \mathrm{m}$ sections were stained for $\alpha$-SMA, Collagen-I (Col-I) and phospho-FAK analyses. Slides were incubated sequentially with anti-Col-I primary antibody (1:1000, Abcam, Hong Kong, China). Then, sections were stained with goat anti-rabbit secondary antibody (ZSGB-BIO, Beijing, China), incubated with 3,3'-diaminobenzidine (ZSGB-BIO, Beijing, China) and evaluated for adequacy using a standard bright field microscope. Anti- $\alpha$-SMA (1:400, Abcam, Hong Kong, China) and anti-phospho-FAK(1:500, Bioworld, 
Shanghai, China) primary antibodies were incubated and pre-labeled with fluorescein (FITC)-conjugated IgG (1:400, Santa Cruz) or rhodamine (TRITC)-conjugated IgG (1:400, Santa Cruz) were used, respectively. Nuclei was stained by 4',6-diamidino-2-phenylindole (DAPI). Slidces were then examinanalyzed under a Leica TCS-SP5 Confocal Laser Scanning Microscope (Leica, Germany).

Total tissue protein was obtained from the border zone of left ventricular myocardial tissues; cellular protein was obtained from the treated cardiac fibroblasts. Tissue or cell lysates were electrophoresed, separated on $10 \%$ or $15 \%$ SDS-PAGE and transferred onto PVDF membranes (Merck Millipore, Billerica, MA, USA). Membranes were blocked with 5\% skim milk at room temperature for one hour; then, incubated overnight on a horizontal shaker at $4{ }^{\circ} \mathrm{C}$ with primary antibodies, including rabbit anti-Akt $(1: 1,000$; CST, Shanghai, China), rabbit anti-phospho-Akt (1:1,000; CST, Shanghai, China), rabbit anti-FAK (1:1,000; CST, Shanghai, China), rabbit anti-phospho-FAK (1:1,000; CST, Shanghai, China), rabbit anti-mTOR (1:1,000; CST, Shanghai, China), rabbit anti- phospho-mTOR (1:1,000; CST, Shanghai, China), rabbit anti-P70S6K (1:1,000; CST, Shanghai, China), rabbit anti- phospho-P70S6K (1:1,000; CST, Shanghai, China), rabbit antiERK1/2 (1:1,000; CST, Shanghai, China), rabbit anti- phospho-ERK1/2 (1:1,000; CST, Shanghai, China) and rabbit anti-GAPDH (1:10,000; CST, Shanghai, China). Membranes were incubated for two hours with goat anti-rabbit antibody (ZSGB-BIO, Beijing, China; 1:5,000 or 1:10,000) stained with ECL detection reagents (Amersham Pharmacia Biotech). Results were detected in a densitometric detection system (Bio-Rad) and expressed as density values normalized to GAPDH.

All data are presented as mean \pm standard deviation (SD). Statistical analysis was performed using One-way ANOVA followed by Bonferroni's test to compare multiple groups, and two-tailed Student's t-test to compare means of the two groups. Statistical analyses were performed using software Graphpad Prism 5.01. Differences with $\mathrm{p}<0.05$ were considered significant.

\section{Results}

A total of 30 mice randomly received surgical treatment; seven mice were used as sham control and 23 mice were for CAL. One week after surgery, 15 mice with CAL treatment survived and were randomly divided into two groups; six mice were orally given PF-562271 (PF group) and nine mice were orally given methylcellulose (as control in MI group).

Four mice died of uncontrollable bleeding or pneumothorax during CAL surgery and two mice died of cardiac rupture or heart failure within the first week of post-surgery, as

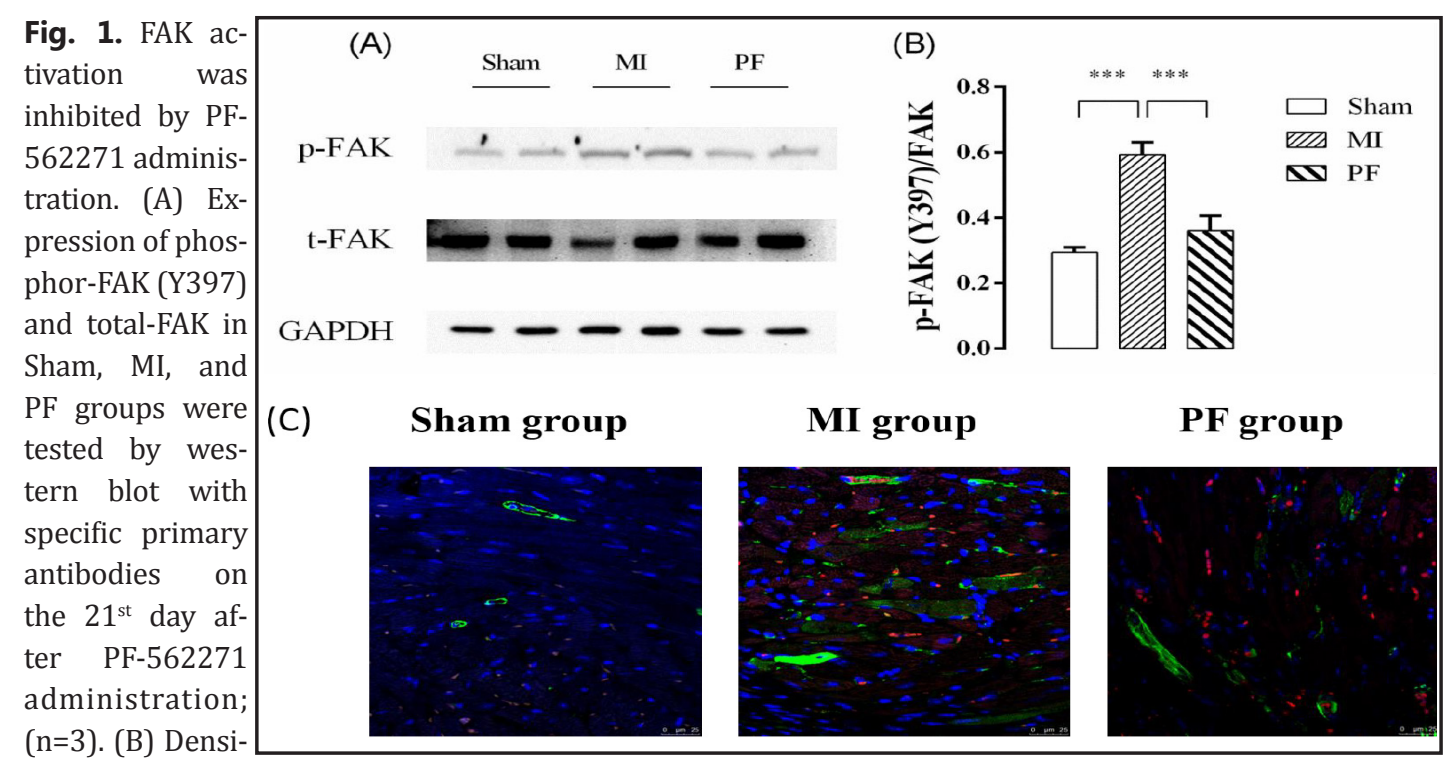

tometric analysis of blots for determining phosphorylated and total protein ratio showed significant increase of phosphor-FAK in the MI group, and was markedly decreased in the PF group; * $\mathrm{p}<0.001$. (C) Anti $\alpha$-SMA (1:400) showed a green-stained and anti-p-FAK (1:500) showed a red-stained in cytoplasm for immunofluorescence. PF-562271 could reduce FAK activation (bar=25 $\mu \mathrm{m}$ ), which can be found in the PF group. 
Table 1. Echocardiographic analysis and tissue harvest in Sham and CAL mice. Values were means of three separate M-mode measurements. MI: myocardial infarction; PF: treated with PF-562271; BW: body weight; HW: heart weight; LW: lung weight; HR: heart rate; LVAW: left ventricular anterior wall; $\mathrm{d}$ : diastole; LVID: left ventricular internal dimension; LVPW: left ventricular posterior wall; \%FS: percent fractional shortening; LV Vol.: left ventricular volu$\mathrm{me} ;{ }^{*} \mathrm{p}<0.05,{ }^{* *} \mathrm{p}<0.01,{ }^{* * *} \mathrm{p}<0.001$

\begin{tabular}{llll}
\hline & $\begin{array}{c}\text { Sham group } \\
(\mathrm{n}=7)\end{array}$ & \multicolumn{1}{c}{$\begin{array}{c}\text { MI group } \\
(\mathrm{n}=6)\end{array}$} & \multicolumn{1}{c}{$\begin{array}{c}\text { PF group } \\
(\mathrm{n}=6)\end{array}$} \\
\hline BW & $20.86 \pm 4.67$ & $24.15 \pm 2.85$ & $22.67 \pm 1.04$ \\
HW & $90.14 \pm 21.26$ & $127.67 \pm 15.92^{* *}$ & $122.83 \pm 15.88$ \\
LW & $113.57 \pm 22.96$ & $142.67 \pm 16.69^{* *}$ & $127.00 \pm 9.47$ \\
HW/BW & $4.32 \pm 0.15$ & $5.29 \pm 0.28^{* * *}$ & $5.41 \pm 0.56$ \\
LW/BW & $5.47 \pm 0.30$ & $6.00 \pm 1.08$ & $5.60 \pm 0.28$ \\
HR & $463.57 \pm 43.37$ & $517.33 \pm 17.34^{*}$ & $491.67 \pm 43.3$ \\
LVAW; d & $0.75 \pm 0.08$ & $0.22 \pm 0.09^{* * *}$ & $0.49 \pm 0.21$ \\
LVID; d & $3.42 \pm 0.37$ & $5.13 \pm 0.86^{* * *}$ & $4.76 \pm 0.81$ \\
LVPW; d & $0.70 \pm 0.13$ & $0.62 \pm 0.08$ & $0.85 \pm 0.52$ \\
\%FS & $31.09 \pm 4.83$ & $15.08 \pm 4.21^{* * *}$ & $18.74 \pm 5.60$ \\
LV Vol.; d & $48.93 \pm 13.20$ & $129.73 \pm 50.76^{*}$ & $109.36 \pm 40.07$ \\
\hline
\end{tabular}

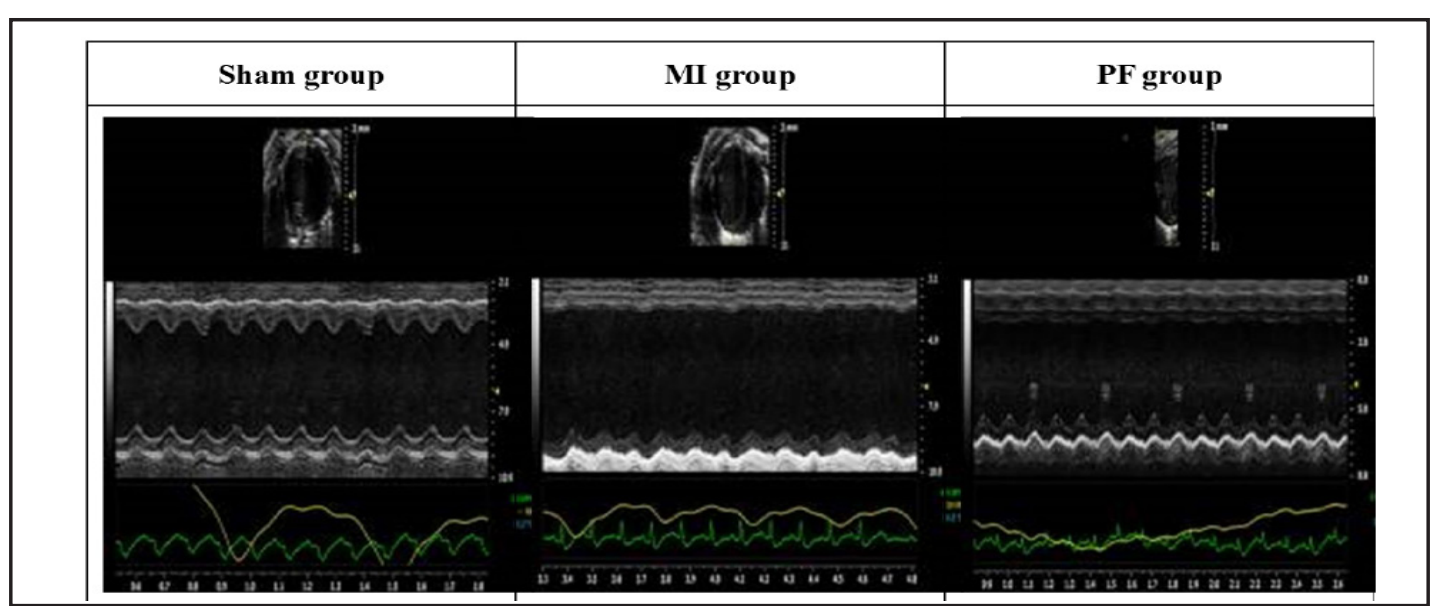

Fig. 2. Echocardiography measurement in experimental groups. Representative M-mode echocardiography of all three groups at the $4^{\text {th }}$ week after Sham or CAL treatment showed increased left ventricular dimension in MI and PF groups, but there was no significant difference between the two groups.

evidenced by autopsy. During pharmacologic administration, two mice died of heart failure in the MI group and none died in the PF group. Heart and body weight ratio of MI mice $(5.29 \pm 0.28)$ significantly increased compared with the sham group $(4.32 \pm 0.15, \mathrm{p}<0.001)$, supporting the establishment of a hypertrophy heart with the MI model.

During the three-week pharmacologic administration period, mice in the MI group (received 0.5\% methylcellulose) and PF group (received PF-562271 mixed with $0.5 \%$ methylcellulose) presented no appearance and life pattern differences. At the end of treatment, heart tissue phosphorylated FAK was analyzed by Western Blot. Results showed a significantly higher expression in the MI group than the Sham group $(\mathrm{p}<0.001)$, and a markedly lower expression in the PF group compared with the MI group ( $\mathrm{p}<0.001)$ (Fig. 1, A and B), indicating the effective inhibition of FAK activation with PF-562271 treatment.

As determined by immunofluorescence staining, FAK could be stimulated through MI. Concurrently, after a 21-day treatment with FAK inhibitor PF-562271, p-FAK expression significantly decreased in both cardiomyocytes and myofibroblasts at of the infarct border zone (Fig. 1C).

At the $4^{\text {th }}$ week post-CAL (after three weeks of pharmacologic administration), echocardiography analysis was carried out for each mouse in all groups (Table 1). Suffering from CAL, mice showed significantly increased heart rates $(\mathrm{p}<0.05)$. Diastolic left ventricular anterior wall (LVAW), left ventricular internal dimension (LVID) and left ventricular volume were significantly different between the MI and control groups $(\mathrm{p}<0.001, \mathrm{p}<0.001, \mathrm{p}<0.05$, 


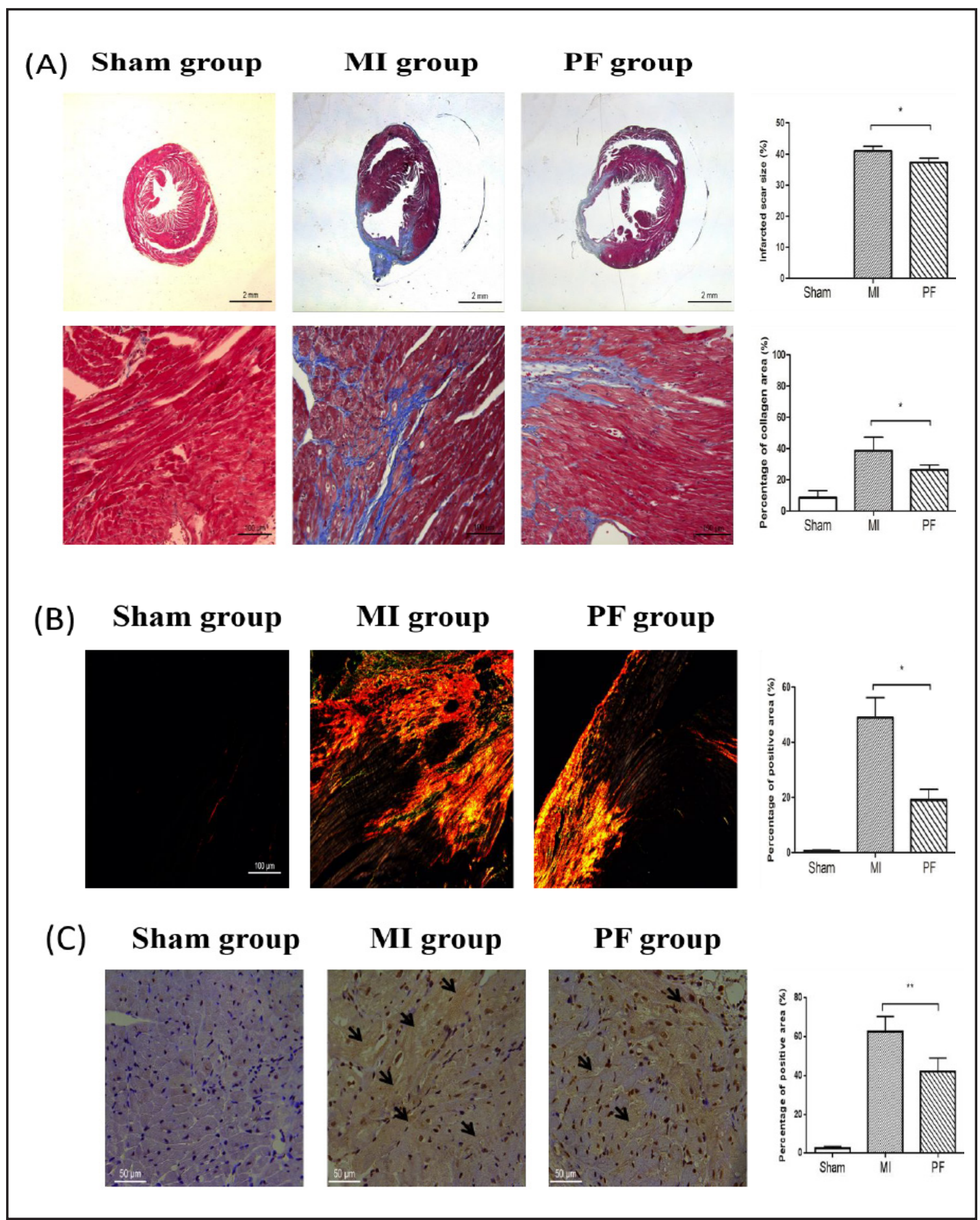

Fig. 3. Cardiac fibrosis changes measured by pathologic staining. (A) Masson's trichrome staining: viable myocardium was stained red, while fibrosis was stained blue due to infarction damage. In the sham operation control group, nearly no area was stained blue. In contrast, blue scar tissues could be obviously seen in the border zone of MI group sections. Then, PF-562271 treatment reduced the area of intensified blue staining ( $n=4$; images were taken at upper row, bar= $2 \mathrm{~mm}$; lower row, bar=100 $\mu \mathrm{m}$ ), and the calculation of collagen area are presented right in the histogram. (B) Sirius red staining: type I collagen performed red to yellow, type III collagen performed green and normal tissue performed gray. More collagens could be seen in the MI group with infiltration to non-fibrotic tissues. In the PF group, amount of red or yellow showed significant decrease $(n=3$; bar=100 $\mu \mathrm{m})$. (C) Immunohistochemistry: with specific primary antibody to Col-I, increased expression of these proteins (black arrow) can be seen at the extracellular matrix in the MI group. PF-562271 could reduce Col-I synthesis (bar=50 $\mu \mathrm{m})$, which was found in the PF group $(\mathrm{n}=3)$.

\section{KARGER}




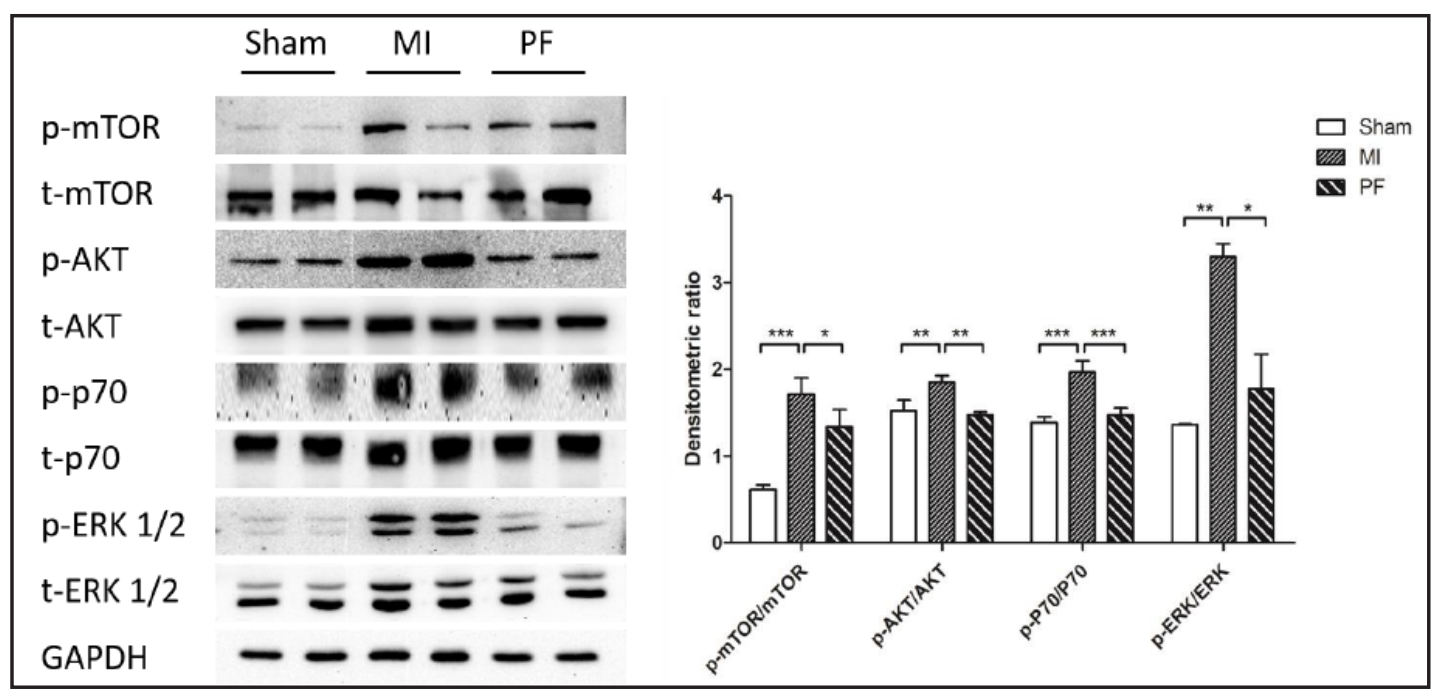

Fig. 4. Regulation of FAK-related signaling pathway analyzed by Western Blot. Expressions of phosphor-mTOR, AKT (S473), P70S6K, ERK1/2 and total- mTOR, AKT (S473), P70S6K, ERK1/2 in Sham, MI, and $\mathrm{PF}$ groups (two individual samples were loaded in each group) were tested by Western Blot with specific primary antibodies on the $21^{\text {st }}$ day after PF-562271 administration ( $\left.n=3\right)$. Densitometric analysis of blots for determining phosphorylated to total protein ratio showed a significant increase of relevant proteins in the MI group, and markedly decreased in the PF group; ${ }^{*} \mathrm{p}<0.05,{ }^{* *} \mathrm{p}<0.01,{ }^{* * *} \mathrm{p}<0.001$.

respectively). As shown in Fig. 2, both $\mathrm{MI}$ and PF groups have thinner anterior walls and an enlarged left ventricle compared with the control group; however, no differences were obtained between the two groups. Surgical treatment did not affect the ventricular posterior wall.

Tissues were harvested and weighted after echocardiography (Table 1). Increased heart or lung mass was shown with CAL (comparing MI group with Sham group, $\mathrm{p}<0.01$ ), especially when heart weight was adjusted with body weight $(\mathrm{p}<0.001)$, indicating deteriorated cardiac function; while FAK inhibition did not bring out any significant change in all aspects of measurements or significant improvement of heart function (comparing PF group with MI group).

At the $4^{\text {th }}$ week of CAL treatment, moderate infarcted area was observed in MI and PF groups by Masson's trichrome staining (Fig. 3A). However, fibrotic tissues significantly increased in the MI group, especially interstitial fibrosis surrounding myocytes. After FAK inhibition, fibrotic tissues at the border zone were fewer in the PF group than in the MI group. Sirius red staining for tissue collagens that reflected at polarized light performance resulted in the same conclusion (Fig. 3B), both of type I and III collagen could be detected in MI group, but less type III collagen in PF group.

Immunohistochemistry analysis has shown a markedly increased expression of Col-I at extracellular matrix at the border zone, comparing the MI group with the control group (Fig. 3C); suggesting that Col-I, a fibrosis condition marker, stimulated synthesis by MI. After 4 -week treatment of FAK inhibitor PF-562271, Col-I expression significantly attenuated $(\mathrm{p}<0.01)$.

CAL surgical treatment significantly increased activated FAK, phosphorylated mTOR, AKT (ser473), P70S6K and ERK1/2 expressions assessed by Western Blot (Fig. 4). However, FAK ( $\mathrm{p}<0.01)$, AKT and P70S6K ( $\mathrm{p}<0.01$ and $\mathrm{p}<0.001$, respectively), and ERK1/2 $(\mathrm{p}<0.05)$ expressions could be suppressed at the $28^{\text {th }}$ day of post-MI pharmacological intervention with PF-562271.

As we previously tested, PP2 could also significantly decrease activation of FAK by hypoxia treatment (Fig. 5A) in vitro. After hypoxia treatment with PP2 intervention, cells were fixed and immunofluorescence stained (Fig. 5B). Hypoxia induced $\alpha$-SMA and vimentin 
(A)

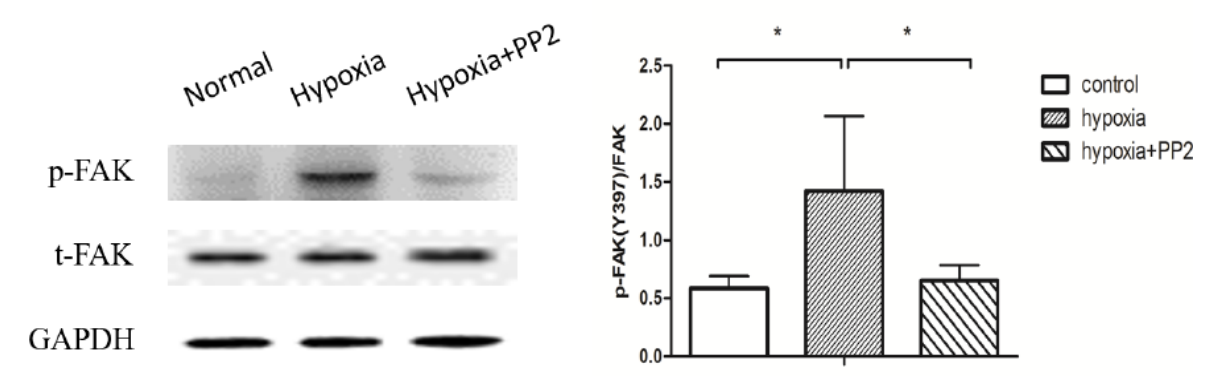

(B) control hypoxia hypoxia+PP2
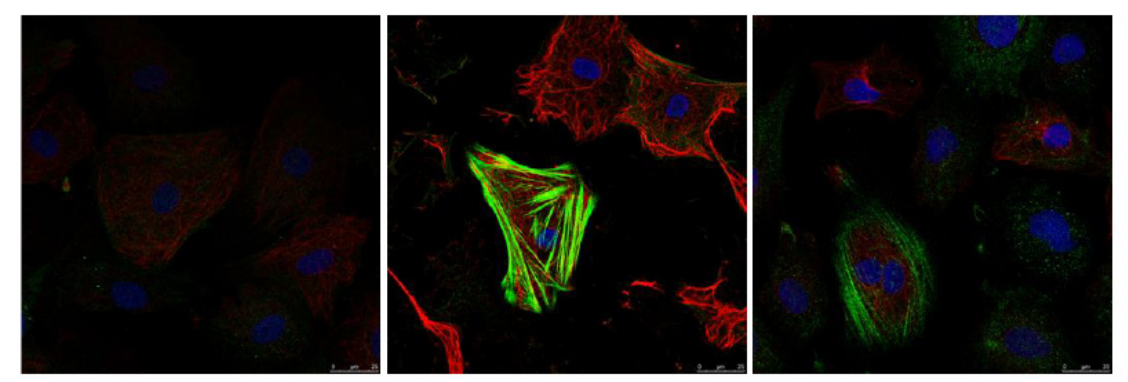

(C)
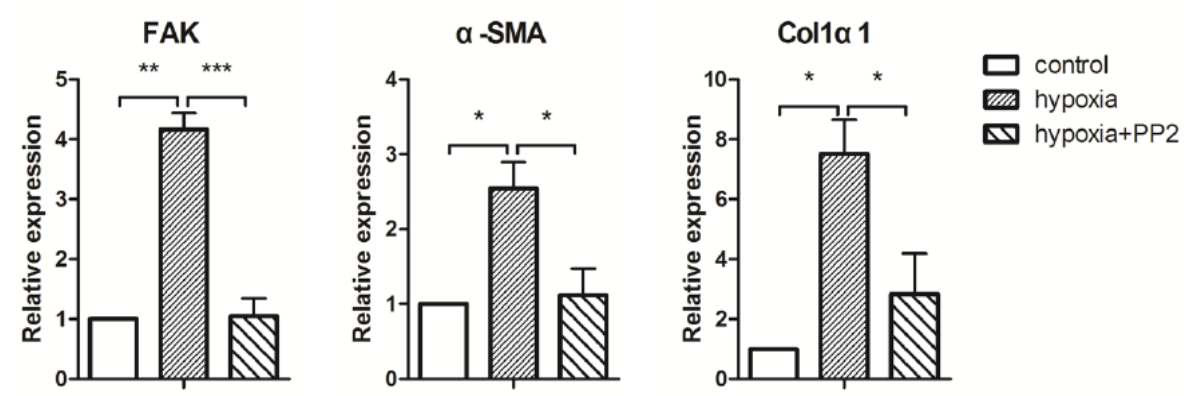

Fig. 5. Inhibition of FAK activation on fibroblast differentiation and collagen synthesis. (A) Expression of phosphor-FAK (Y397) and total-FAK in the control, hypoxia, and hypoxia+PP2 groups were tested by Wes ${ }^{-}$ tern blot with specific primary antibodies after PP2 treatment in cardiac fibroblasts; $(n=3)$. (B) Immunoflu ${ }^{-}$ orescence expression of cardiac fibroblasts. Fibroblasts treated with anti $\alpha$-SMA showed a blue-stained nucleus and anti-vimentin showed a red-stained cytoplasm, after exposure to hypoxia with or without PP2 treatment for 24 hours (bar=25 $\mu \mathrm{m}$ ). (C) RT-PCR analysis on cardiac fibroblasts: note that 24-hour hypoxia treatment significantly induced high FAK, $\alpha$-SMA and Col1 $\alpha 1$ expressions. FAK inhibition with PP2 markedly decreased this induction; ${ }^{*} \mathrm{p}<0.05,{ }^{* *} \mathrm{p}<0.01,{ }^{* * *} \mathrm{p}<0.001$.

expressions, indicating that fibroblast differentiation occurred. However, their expressions were reduced by FAK inhibition with PP2; which was indicated by lower fluorescence intensity in the hypoxia+PP2 group than in the hypoxia group. FAK, $\alpha$-SMA and Col1 $\alpha 1$ gene expressions were analyzed by RT-PCR; and revealed that expressions in both hypoxia and hypoxia+PP2 groups under hypoxia increased compared with the control group. PP2 treatment could significantly suppress FAK $(p<0.001), \alpha-S M A(p<0.05)$ and $\operatorname{Col} 1 \alpha 1(p<0.05)$ RNA expressions (Fig. 5C). 


\section{Discussion}

Previous studies, including ours $[10,13,14]$, indicated that FAK is important in cardiac fibrosis formation. Herein, we developed a strategy to target FAK by pharmacological intervention and demonstrated that transient FAK inhibition can attenuate cardiac fibrosis by inducing a 4 -week CAL on mice models.

It is well known that FAK is dispensable for basal myocyte function and mediates fibrosis response in various diseases; several studies demonstrated a beneficial consequence by inhibiting its expression under pathological conditions [17-19].

However, the role for FAK in cardiac fibrosis is still controversial. Research is mainly carried out in myocyte restricted knockout mice or small interfering RNA transient silencing; Peng et al. and DiMichele et al. both used FAK myocyte restricted knockout mice in their reports $[8,20]$. At cardiac hypertrophy conditions, FAK deletion in cardiac myocyte could predispose a premature maladaptive remodeling with increased cardiac fibrosis. In Dr. Taylor's laboratory $[9,12]$, no significant changes were found on cardiac fibrosis in I/R models, either on FAK activation or inactivation treatment solely in cardiomyocytes. However, prediction of relational pathological fibrosis and detrimental cardiac remodeling was evident even when FAK was knocked out strictly in cardiomyocytes. On the contrary, comprehensive effects were demonstrated by Dr. Clemente and colleagues [10], revealing the fact that myocardial FAK silencing, in contrast to myocyte-restricted knockout, may attenuate and even reverse fibrosis development in a load-induced cardiac hypertrophy mice model, demonstrating that FAK inhibition in global area of the heart may play a vital role in that condition [15].

Given the pivotal role of FAK in fibroblast activation, pharmaceutically targeting FAK actions may be feasible in counteracting fibrosis. Based on our previous discoveries [19], in this present study, we developed a hypoxic model and tested it on CFs. Cell differentiation was markedly stimulated under ischemic and hypoxic conditions, demonstrated by high $\alpha$-SMA and vimentin expressions; and FAK inhibition with PP2 could suppress this phenotype alteration, as well as collagen synthesis. Among pharmaceutical options, PF-562271 has been shown to effectively inhibit FAK activation in vivo [21-23]. Our results are fully in agreement with the results demonstrated by Pascual et al. [19]; wherein, pharmacologic FAK inhibition by PF-562271 could suppresse the activation of FAK in both cardiomyocytes and myofibroblasts, and attenuate cardiac fibrosis post-MI, paralleling with down-regulation of its relevant signaling pathways, including mTOR, AKT/ P70S6K and ERK1/2 (Fig. 6).

We previously reported that AKT/S6k was involved in CFs differentiation induced by TGF- $\beta$ stimulation in vitro, and not the ERK1/2 signaling pathway; which was confirmed by FAK inhibition with FAK/Src kinase inhibitor PP2 and other related inhibitors [14]. This current in vivo study has shown an unexpected result; wherein, both AKT/S6k signaling and ERK1/2 participated in this procedure, in addition to mTOR involvement [24]. We believe that a more complex environment exists in vivo, cell differentiation may not be solely stimulated by TGF- $\beta$, and other mechanisms could also influence relevant protein expressions in MI animal models. This present study might contribute to our new knowledge on cellular pathway activation.

It is interesting that associated heart functions did not show significant improvement, even though FAK inhibition resulted in a reduced level of fibrosis post-MI. Cardiac remodeling is well known to be developed in time and in a special-dependent basis [1]; which is closely associated with collagen accumulation, as well as a balance between collagen synthesis and degradation, both in infarcted and non-infarcted myocardium. In this present study, increased collagen expressions in the MI process was evidenced, which can be a response to ischemia and hypoxia; while FAK inhibition could suppress collagen expressions and fibroblast differentiation [25]. Heart function usually deteriorates with MI progression; however, 21 days of FAK inhibition might not be able to sufficiently attenuate or reverse interstitial fibrosis, as previously suggested [10]. Herein, it may be expected that a longer intervention could bring in more beneficial outcomes that show heart function recovery. In addition to 
Fig. 6. Schematic representation of FAK involvement in singling pathways. FAK can be activated by phosphorylation through multiple profibrotic factors. Its downstream PI3K/AKT pathway is then activated through phosphorylation at the Tyr397 site, followed by mTOR/P70S6K activation. JNK/ ERK related MAPK pathway can also be activated simultaneously by FAK with phosphorylation at Tyr925, followed by activation of the ERK1/2 pathway. Either mTOR/P70S6K or ERK1/2 or both can induce $\alpha$-SMA-positive myofibroblast differentiation together

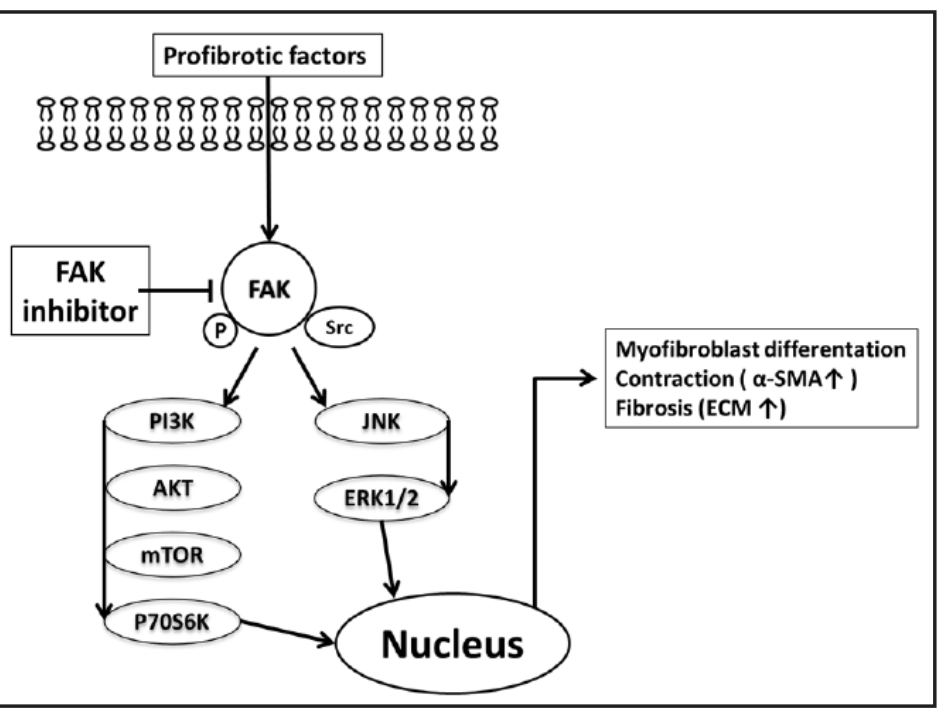
with synthesis of different collagen types, reflecting fibrosis development. Consequently, this response could be suppressed by typical FAK inhibitors.

our discoveries from the present study, we have established a moderate infarction model, which triggered fibrotic processes without seriously damaging heart function. This model may have potential for studying the efficacy of pharmaceutical FAK inhibition in improving heart function after MI.

The main limitation of this study may be lack of the group of Sham+PF-562,271, although it has been declared to be innocuous to normal organs [26], and a relatively short period of time was chosen for the study, as the course of MI involves complicated mechanisms with multiple regulators. We might also not be able to obtain more sophisticated cardiac measurements due to limited functionality of the ultrasound instrument used in the experiments. A study with a longer time period that focuses on different time points is currently being undertaken in our laboratory to uncover diverse connections among relevant proteins involved in signaling pathways.

FAK functions as a mediator in cardiac fibrosis post-MI and is involved in many signaling pathways (Fig. 6). By pharmacologically targeting inhibition of PF-562271, cardiac fibrosis could be attenuated within a 4-week observation post-MI; although heart function did not significantly improve during the period. Both AKT/P70S6k and ERK1/2 signaling pathways are involved in CFs differentiation, and these results support the notion that FAK inhibition would be an optional strategy to attenuate fibrosis post-MI, and its beneficial role still need to be explored.

\section{Acknowledgments}

The authors thank Ms. Qing Xu of Capital Medical University, Beijing, China for providing a sincere echocardiography measurement. We also thank Dr. Pascual of Universidad Autónoma de Madrid, Madrid, Spain for giving us critical suggestions on pharmacological administration.

Contract grant sponsor: the National Natural Science Foundation of China; Contract grant number: 81470423 . 


\section{Disclosure Statement}

None declared.

\section{References}

1 Sutton MG, Sharpe N: Left ventricular remodeling after myocardial infarction: Pathophysiology and therapy. Circulation 2000;101:2981-2988.

2 Dean RG, Balding LC, Candido R, Burns WC, Cao Z, Twigg SM, Burrell LM: Connective tissue growth factor and cardiac fibrosis after myocardial infarction. J Histochem Cytochem 2005;53:1245-1256.

3 Weber KT: Extracellular matrix remodeling in heart failure: A role for de novo angiotensin II generation. Circulation 1997;96:4065-4082.

4 Parsons JT: Focal adhesion kinase: The first ten years. J Cell Sci 2003;116:1409-1416.

5 McLean GW, Carragher NO, Avizienyte E, Evans J, Brunton VG, Frame MC: The role of focal-adhesion kinase in cancer - a new therapeutic opportunity. Nat Rev Cancer 2005;5:505-515.

6 Cohen LA, Guan JL: Mechanisms of focal adhesion kinase regulation. Curr Cancer Drug Targets 2005;5:629643.

7 Lagares D, Kapoor M: Targeting focal adhesion kinase in fibrotic diseases. BIODRUGS 2013;27:15-23.

8 DiMichele LA, Doherty JT, Rojas M, Beggs HE, Reichardt LF, Mack CP, Taylor JM: Myocyte-restricted focal adhesion kinase deletion attenuates pressure overload-induced hypertrophy. Circ Res 2006;99:636-645.

9 Hakim ZS, DiMichele LA, Rojas M, Meredith D, Mack CP, Taylor JM: FAK regulates cardiomyocyte survival following ischemia/reperfusion. J Mol Cell Cardiol 2009;46:241-248.

10 Clemente CF, Tornatore TF, Theizen TH, Deckmann AC, Pereira TC, Lopes-Cendes I, Souza JR, Franchini KG: Targeting focal adhesion kinase with small interfering RNA prevents and reverses load-induced cardiac hypertrophy in mice. Circ Res 2007;101:1339-1348.

11 Liang W, Ren K, Liu F, Cui W, Wang Q Chen Z, Fan W: Periodic mechanical stress stimulates the FAK mitogenic signal in rat chondrocytes through ERK1/2 activity. CELL PHYSIOL BIOCHEM 2013;32:915-930.

12 Cheng Z, DiMichele LA, Hakim ZS, Rojas M, Mack CP, Taylor JM: Targeted focal adhesion kinase activation in cardiomyocytes protects the heart from ischemia/reperfusion injury. Arterioscler Thromb Vasc Biol 2012;32:924-933.

13 Zhang P, Wang W, Wang X, Wang X, Song Y, Han Y, Zhang J, Zhao H: Protein analysis of atrial fibrosis via label-free proteomics in chronic atrial fibrillation patients with mitral valve disease. PLOS ONE 2013;8:e60210.

14 Zhang P, Wang W, Wang X, Wang X, Song Y, Zhang J, Zhao H: Focal adhesion kinase mediates atrial fibrosis via the AKT/S6K signaling pathway in chronic atrial fibrillation patients with rheumatic mitral valve disease. Int J Cardiol 2013;168:3200-3207.

15 Moore-Morris T, Guimaraes-Camboa N, Banerjee I, Zambon AC, Kisseleva T, Velayoudon A, Stallcup WB, Gu Y, Dalton ND, Cedenilla M, Gomez-Amaro R, Zhou B, Brenner DA, Peterson KL, Chen J, Evans SM: Resident fibroblast lineages mediate pressure overload-induced cardiac fibrosis. J Clin Invest 2014;124:2921-2934.

16 Gong X, Fan G, Wang W, Wang G: Trimetazidine protects umbilical cord mesenchymal stem cells against hypoxia and serum deprivation induced apoptosis by activation of Akt. Cell Physiol Biochem 2014;34:2245-2255.

17 Infusino GA, Jacobson JR: Endothelial FAK as a therapeutic target in disease. Microvasc Res 2012;83:89-96.

18 Hong S, Lee JB, Iizuka Y, Song YK, Seong GJ, Han SH: The role of focal adhesion kinase in the TGF-betainduced myofibroblast transdifferentiation of human Tenon's fibroblasts. Korean J Ophthalmol 2012;26:4548.

19 Lagares D, Busnadiego O, Garcia-Fernandez RA, Kapoor M, Liu S, Carter DE, Abraham D, Shi-Wen X, Carreira P, Fontaine BA, Shea BS, Tager AM, Leask A, Lamas S, Rodriguez-Pascual F: Inhibition of focal adhesion kinase prevents experimental lung fibrosis and myofibroblast formation. Arthritis Rheum 2012;64:16531664.

20 Peng X, Kraus MS, Wei H, Shen TL, Pariaut R, Alcaraz A, Ji G, Cheng L, Yang Q Kotlikoff MI, Chen J, Chien $\mathrm{K}, \mathrm{Gu} \mathrm{H}$, Guan JL: Inactivation of focal adhesion kinase in cardiomyocytes promotes eccentric cardiac hypertrophy and fibrosis in mice. J Clin Invest 2006;116:217-227. 


\section{Cellular Physiology Cell Physiol Biochem 2015;37:515-526}

\begin{tabular}{l|l|l|}
\hline DOI: 10.1159/000430373 & (0) 2015 S. Karger AG, Basel \\
\hline
\end{tabular}

Fan et al.: A Promising Target to Cardiac Fibrosis

21 Bagi CM, Christensen J, Cohen DP, Roberts WG, Wilkie D, Swanson T, Tuthill T, Andresen CJ: Sunitinib and PF-562,271 (FAK/Pyk2 inhibitor) effectively block growth and recovery of human hepatocellular carcinoma in a rat xenograft model. Cancer Biol Ther 2009;8:856-865.

22 Bagi CM, Roberts GW, Andresen CJ: Dual focal adhesion kinase/Pyk2 inhibitor has positive effects on bone tumors: Implications for bone metastases. Cancer 2008;112:2313-2321.

23 Roberts WG, Ung E, Whalen P, Cooper B, Hulford C, Autry C, Richter D, Emerson E, Lin J, Kath J, Coleman K, Yao L, Martinez-Alsina L, Lorenzen M, Berliner M, Luzzio M, Patel N, Schmitt E, LaGreca S, Jani J, Wessel M, Marr E, Griffor M, Vajdos F: Antitumor activity and pharmacology of a selective focal adhesion kinase inhibitor, PF-562,271. Cancer Res 2008;68:1935-1944.

24 Dalla CA, Clemente CF, Carvalho HF, Carvalheira JB, Nadruz WJ, Franchini KG: FAK mediates the activation of cardiac fibroblasts induced by mechanical stress through regulation of the mTOR complex. Cardiovasc Res 2010;86:421-431.

25 Greenberg RS, Bernstein AM, Benezra M, Gelman IH, Taliana L, Masur SK: FAK-dependent regulation of myofibroblast differentiation. FASEB J 2006;20:1006-1008.

26 Roberts WG, Ung E, Whalen P, Cooper B, Hulford C, Autry C, Richter D, Emerson E, Lin J, Kath J, Coleman K, Yao L, Martinez-Alsina L, Lorenzen M, Berliner M, Luzzio M, Patel N, Schmitt E, LaGreca S, Jani J, Wessel M, Marr E, Griffor M, Vajdos F: Antitumor activity and pharmacology of a selective focal adhesion kinase inhibitor, PF-562,271. Cancer Res 2008;68:1935-1944. 